\title{
Reviewer acknowledgment 2015
}

Vipin $Z_{\text {amvar }}{ }^{* *}$ and David Taggart ${ }^{2}$

\section{Contributing reviewers}

The Editors of the Journal of Cardiothoracic Surgery would like to thank all of our reviewers who have contributed to the journal in Volume 10 (2015) and whose valuable support is fundamental to its success.

\author{
Ahmad Abugameh \\ Germany \\ Anders Ahlsson \\ Sweden

\section{Aslı Gül Akgül} \\ Turkey \\ Rui Almeida \\ Brazil
}

Arangannal Arul

UK

Conal Austin

UK

Tara Baetz

Canada

Clifford Barlow

UK

Volkan Baysungur

Turkey

\section{Pascal Berna}

France

Jose M Bernal

Spain

\section{Alessandro Bertani \\ Italy}

Timothy Billiar

USA

Nikolaos Bonaros

Austria

\author{
Allison Cabalka \\ USA
}

Huseyin Altug Cakmak

Turkey

\section{Antonio Calafiore}

Saudi Arabia

Leonardo Canale

Brazil

Huiqing Cao

China

Burçin Çelik

Turkey

\section{Liangwan Chen}

China

Zhenguang Chen

China

Paola Ciriaco

Italy

\section{Francisco Costa \\ Brazil}

Abhishek Deshmukh

USA

\section{Alpha Diallo \\ UK}

Doa El-Ansary

Australia

Bachar El Oumeiri

Belgium

\author{
Wentao Fang \\ China \\ Yue Feng \\ China
}

Christophoros Foroulis

Greece

Changqing Gao

China

Roman Gottardi

Austria

Jibo Hu

China

Shengshou Hu

China

Antonio Lio

Italy

Changyu Liu

Chile

Alan Kirk

Valerie Lacroix

Belgium

Yongqiang Lai

China

Harry Lapierre

Canada

Zhidong Liu

China

* Correspondence: cardiothoracicsurgery@biomedcentral.com

${ }^{1}$ Royal Infirmary of Edinburgh, Edinburgh, UK

Full list of author information is available at the end of the article 
Haiyan Lou

China

Donna Maziak

Canada

John Moore

Australia

Henrique Murad

Brazil

Girish Nair

Canada

Vinicius Nina

Brazil

Robert Nolan

Canada

Maral Ouzounian

Canada

Bharat Pancholy

USA

Jason Peart

Australia

Mate Petricevic

Croatia

Claudio Picariello

Italy
Edvin Prifti

Albania

Eduardo Rocha

Brazil

Alfredo Rodrigues

Brazil

Alistair Royse

Australia

Fernando Sales

Brazil

Cleusa Santos

Brazil

Thomas Schachner

Austria

Pedro Silva

Brazil

James Tatoulis

Australia

Euclides Tenorio

Brazil

Pierre Voisine

Canada

Song Wan

China
Bing Yen Wang

Taiwan

Qing Wang

China

Xi Ming Wang

China

Atsushi Watanabe

Japan

Guangzhao Yang

China

Mohammed Yasin

UK

Can Yerebakan

Germany

Hong Yu

China

Author details

${ }^{1}$ Royal Infirmary of Edinburgh, Edinburgh, UK.

${ }^{2}$ John Radcliffe Hospital, Oxford, UK. 